\title{
First-Order Linear-time Epistemic Logic with Group Knowledge: An Axiomatisation of the Monodic Fragment
}

\author{
Francesco Belardinelli and Alessio Lomuscio \\ Department of Computing \\ Imperial College London, UK \\ $\{$ F.Belardinelli,A.Lomuscio\}@imperial.ac.uk
}

\begin{abstract}
We investigate quantified interpreted systems, a computationally grounded semantics for a first-order temporal epistemic logic on linear time. We report a completeness result for the monodic fragment of a language that includes LTL modalities as well as distributed and common knowledge. We exemplify possible uses of the formalism by analysing message passing systems, a typical framework for distributed systems, in a first-order setting.
\end{abstract}

\section{Introduction}

Propositional modal logics to reason about knowledge and time have been thoroughly investigated by researchers in artificial intelligence both as regards their theoretical properties (completeness, decidability, complexity) [7,9], as well as for the specification and verification of multi-agent systems [4, 28].

These temporal epistemic logics have been explored in several directions. In one line of research, epistemic modalities have been added to represent group knowledge such as distributed and common knowledge $[8,10]$. In another one, the temporal fragment has been modified according to different models of time (e.g., linear or branching, discrete or continuous) [16,18]. In yet another line, temporal epistemic logic has been studied at the first order $[1,15]$.

In this paper we extend a combination of epistemic and temporal logic to the predicate level. We provide this language with a computationally grounded semantics [27] given in terms of quantified interpreted systems [1,2], and we present a complete axiomatisation of the monodic fragment of this logic, where at most one free variable appears in the scope of any modal operator. Finally, we apply this formalism to the modeling of message passing systems, a typical framework in distributed systems $[17,4]$.

Our starting point is a number of results by Hodkinson, Wolter, and Zakharyaschev, among others, regarding the axiomatisability [22, 25], decidability $[15,24]$, and complexity $[12,13]$ of first-order modal logics, including both positive $[11,21]$ and negative results $[14,23,26]$. Specifically, we prove completeness for our first-order temporal epistemic logic via quasimodels. These structures 
have been used in [15] to prove decidability for monodic fragments of first-order temporal logic (FOTL) on a variety of flows of time. These investigations were further pursued in [14], where branching flows of time are analyzed, and in [11], which deals with the packed fragment of FoTL. In $[12,13]$ the complexity of the decision problem for a number of monodic fragments of FoTL is considered.

As regards general first-order modal logic, the decidability of monodic fragments has been investigated in [24]. In [26] it is proved that first-order epistemic logic with common knowledge is not axiomatisable. However, in [23] it is shown that its monodic fragment is. Finally, this paper relies on results in [22, 25]. In [25] the authors present a complete axiomatisation for the monodic fragment of FoTL on the naturals. In [22] we have a similar result for a variety of first-order epistemic logics with common knowledge. Note that none of these articles uses interpreted systems $[4,19]$ as the underlying semantics, as we do here.

Our motivation for this contribution comes from an interest in first-order temporal epistemic formalisms to model high-level properties of multi-agent systems (MAS). While temporal epistemic logics are well understood at the propositional level, their usefulness has been demonstrated in a number of applications, and model checking tools have been developed for them $[6,20]$, still we believe there is a growing need in web-services, security, as well as other areas, to extend these languages to the first order. As preliminary contributions to this project, in [2] we introduced a "static" version of quantified interpreted systems to model a firstorder epistemic formalism. This was then extended to the temporal dimension in [1]. Differently from these previous works, here we explicitly assume linear-time operators and the natural numbers as the flow of time. Both features are crucial for applications, but they also increase the complexity of the formalism.

Scheme of the paper. In Section 2 we introduce the first-order temporal epistemic language $\mathcal{L}_{m}$, for a set $A=\{1, \ldots, m\}$ of agents, and in Section 3 we provide it with a semantics in terms of quantified interpreted systems and present its monodic fragment. In Section 4 we explore its expressive power in specifying message passing systems. In Sections 5 and 6 we introduce an axiomatisation for the monodic fragment of $\mathcal{L}_{m}$ and prove its completeness. We present detailed proofs in Appendix A.

\section{Syntax}

The first-order temporal epistemic language $\mathcal{L}_{m}$ contains individual variables $x_{1}, x_{2}, \ldots$, individual constants $c_{1}, c_{2}, \ldots$, and $n$-ary predicative letters $P_{1}^{n}, P_{2}^{n}, \ldots$, for $n \in \mathbb{N}$, the propositional connectives $\neg$ and $\rightarrow$, the universal quantifier $\forall$, the temporal operators $\bigcirc$ and $\mathcal{U}$, the epistemic operators $K_{i}$, for $i \in A, D$, and $C$. The only terms $t_{1}, t_{2}, \ldots$ in $\mathcal{L}_{m}$ are individual variables and constants.

Definition 1. Formulas in $\mathcal{L}_{m}$ are defined in the BN form as follows:

$$
\phi::=P^{k}\left(t_{1}, \ldots, t_{k}\right)|\neg \psi| \psi \rightarrow \psi^{\prime}|\forall x \psi| \bigcirc \psi\left|\psi \mathcal{U} \psi^{\prime}\right| K_{i} \psi|D \psi| C \psi
$$

The formulas $\bigcirc \phi$ and $\phi \mathcal{U} \phi^{\prime}$ are read as " $\phi$ holds at the next step" and " $\phi$ ' will hold and $\phi$ is the case until that moment". The formula $K_{i} \phi$ represents 
"agent $i$ knows $\phi$ ", while formulas $D \phi$ and $C \phi$ respectively mean " $\phi$ is distributed knowledge" and " $\phi$ is common knowledge" in the group $A$ of agents.

We define the symbols $\wedge, \vee, \leftrightarrow, \exists, G$ (always in the future), $F$ (some time in the future) as standard. Further, $E \phi=\bigwedge_{i \in A} K_{i} \phi$, and for $\triangle$ equal to $E$ or $\bigcirc, \triangle^{k} \phi$ is defined as follows for $k \in \mathbb{N}: \triangle^{0} \phi=\phi$ and $\triangle^{k+1} \phi=\triangle \triangle^{k} \phi$.

By $\phi[\boldsymbol{y}]$ we mean that $\boldsymbol{y}=y_{1}, \ldots, y_{n}$ are all the free variables in $\phi$; while $\phi[\boldsymbol{y} / \boldsymbol{t}]$ is the formula obtained by substituting simultaneously some, possibly all, free occurrences of $\boldsymbol{y}$ in $\phi$ with $\boldsymbol{t}=t_{1}, \ldots, t_{n}$, renaming bounded variables.

\section{Quantified Interpreted Systems}

In this section we present a dynamic version of the "static" quantified interpreted systems in [2] by assuming the natural numbers $\mathbb{N}$ as the underlying flow of time. Specifically, for each agent $i \in A$ in a multi-agent system we introduce a set $L_{i}$ of local states $l_{i}, l_{i}^{\prime}, \ldots$, and a set $A c t_{i}$ of actions $\alpha_{i}, \alpha_{i}^{\prime}, \ldots$. We consider local states and actions for the environment $e$ as well. The set $\mathcal{S} \subseteq L_{e} \times L_{1} \times \ldots \times L_{m}$ contains the global states of the MAS, while $A c t \subseteq A c t_{e} \times A c t_{1} \times \ldots \times A c t_{m}$ is the set of joint actions. We also introduce a transition function $\tau: A c t \rightarrow(\mathcal{S} \rightarrow \mathcal{S})$. Intuitively, $\tau(\alpha)(s)=s^{\prime}$ encodes that agents can access the global state $s^{\prime}$ from $s$ by performing the joint action $\alpha$. We say that the global state $s^{\prime}$ is reachable in one step from $s$, or $s \sqsubset s^{\prime}$, iff there is $\alpha \in$ Act such that $\tau(\alpha)(s)=s^{\prime}$.

To represent the temporal evolution of the MAS we consider the flow of time $\langle\mathbb{N},<\rangle$ of natural numbers $\mathbb{N}$ with the strict total order $<$. A run $r$ over $\langle\mathcal{S}$, Act, $\tau, \mathbb{N}\rangle$ is a function from $\mathbb{N}$ to $\mathcal{S}$ such that $r(n) \sqsubset r(n+1)$. Intuitively, a run represents a possible evolution of the MAS according to the transition function $\tau$ and assuming $\mathbb{N}$ as the flow of time. We now define the quantified interpreted systems for the language $\mathcal{L}_{m}$ as follows:

Definition 2 (QIS). A quantified interpreted system over $\langle\mathcal{S}$, Act, $\tau, \mathbb{N}\rangle$ is a triple $\mathcal{P}=\langle\mathcal{R}, \mathcal{D}, I\rangle$ such that (i) $\mathcal{R}$ is a non-empty set of runs over $\langle\mathcal{S}$, Act, $\tau, \mathbb{N}\rangle$; (ii) $\mathcal{D}$ is a non-empty set of individuals; (iii) $I$ is an interpretation of $\mathcal{L}_{m}$ such that $I(c) \in \mathcal{D}$, and for $r \in \mathcal{R}, n \in \mathbb{N}, I\left(P^{k}, r, n\right)$ is a $k$-ary relation on $\mathcal{D}$. We denote by $\mathcal{Q I S}$ the class of all quantified interpreted systems.

Following standard notation [4] a pair $(r, n)$ is a point in $\mathcal{P}$. If $r(n)=$ $\left\langle l_{e}, l_{1}, \ldots, l_{m}\right\rangle$ is the global state at the point $(r, n)$, then $r_{e}(n)=l_{e}$ and $r_{i}(n)=l_{i}$ are the environment's and agent $i$ 's local state at $(r, n)$ respectively. Further, a QIS is synchronous if for all $i \in A, r_{i}(n)=r_{i}^{\prime}\left(n^{\prime}\right)$ implies $n=n^{\prime}$, that is, time is part of the local state of any agent. $\mathcal{Q} \mathcal{I} \mathcal{S}^{\text {sync }}$ is the class of all synchronous QIS.

Now we assign a meaning to the formulas of $\mathcal{L}_{m}$ in quantified interpreted systems. Let $\sigma$ be an assignment from the variables to the individuals in $\mathcal{D}$, the valuation $I^{\sigma}(t)$ of a term $t$ is defined as $\sigma(y)$ for $t=y$, and $I^{\sigma}(t)=I(c)$, for $t=c$. A variant $\sigma\left(\begin{array}{l}x \\ a\end{array}\right)$ of an assignment $\sigma$ assigns $a \in \mathcal{D}$ to $x$ and coincides with $\sigma$ on all the other variables.

Definition 3. The satisfaction relation $=$ for $\phi \in \mathcal{L}_{m},(r, n) \in \mathcal{P}$, and an assignment $\sigma$ is defined as follows: 


$$
\begin{aligned}
& \left(\mathcal{P}^{\sigma}, r, n\right) \models P^{k}\left(t_{1}, \ldots, t_{k}\right) \\
& \left(\mathcal{P}^{\sigma}, r, n\right) \models \neg \psi \\
& \left(\mathcal{P}^{\sigma}, r, n\right) \models \psi \rightarrow \psi^{\prime} \\
& \left(\mathcal{P}^{\sigma}, r, n\right) \models \forall x \psi \\
& \left(\mathcal{P}^{\sigma}, r, n\right) \models \bigcirc \psi \\
& \left(\mathcal{P}^{\sigma}, r, n\right) \models \psi \mathcal{U} \psi^{\prime} \\
& \left(\mathcal{P}^{\sigma}, r, n\right) \models K_{i} \psi \\
& \left(\mathcal{P}^{\sigma}, r, n\right) \models D \psi \\
& \left(\mathcal{P}^{\sigma}, r, n\right) \models C \psi
\end{aligned}
$$

$$
\begin{array}{ll}
\text { iff } & \left\langle I^{\sigma}\left(t_{1}\right), \ldots, I^{\sigma}\left(t_{k}\right)\right\rangle \in I\left(P^{k}, r, n\right) \\
\text { iff } & \left(\mathcal{P}^{\sigma}, r, n\right) \not \models \psi \\
\text { iff } & \left(\mathcal{P}^{\sigma}, r, n\right) \not \models \psi \text { or }\left(\mathcal{P}^{\sigma}, r, n\right) \models \psi^{\prime} \\
\text { iff } & \text { for all } a \in \mathcal{D},\left(\mathcal{P}^{\sigma}\left(\begin{array}{l}
x \\
a
\end{array}\right), r, n\right) \models \psi \\
\text { iff } & \left(\mathcal{P}^{\sigma}, r, n+1\right) \models \psi \\
\text { iff } \quad \text { there is } n^{\prime} \geq n \text { such that }\left(\mathcal{P}^{\sigma}, r, n^{\prime}\right) \models \psi^{\prime} \\
& \text { and for all } n^{\prime \prime}, n \leq n^{\prime \prime}<n^{\prime} \text { implies }\left(\mathcal{P}^{\sigma}, r, n^{\prime \prime}\right) \models \psi \\
\text { iff } & \text { for all }\left(r^{\prime}, n^{\prime}\right), r_{i}(n)=r_{i}^{\prime}\left(n^{\prime}\right) \text { implies }\left(\mathcal{P}^{\sigma}, r^{\prime}, n^{\prime}\right) \models \psi \\
\text { iff } & r_{i}(n)=r_{i}^{\prime}\left(n^{\prime}\right) \text { for all } i \in A, \text { implies }\left(\mathcal{P}^{\sigma}, r^{\prime}, n^{\prime}\right) \models \psi \\
\text { iff } & \text { for all } k \in \mathbb{N},\left(\mathcal{P}^{\sigma}, r, n\right) \models E^{k} \psi
\end{array}
$$

The truth conditions for $\wedge, \vee, \leftrightarrow, \exists, G$, and $F$ are defined from those above. A formula $\phi \in \mathcal{L}_{m}$ is true at a point $(r, m)$ iff it is satisfied at $(r, m)$ by every $\sigma$; $\phi$ is valid on a $Q I S \mathcal{P}$ iff it is true at every point in $\mathcal{P} ; \phi$ is valid on a class $\mathcal{C}$ of $Q I S$ iff it is valid on every QIS in $\mathcal{C}$.

\subsection{The monodic fragment}

In what follows we focus on the monodic fragment of the language $\mathcal{L}_{m}$.

Definition 4. The monodic fragment $\mathcal{L}_{m}^{1}$ is the set of formulas $\phi \in \mathcal{L}_{m}$ such that any subformula of $\phi$ of the form $K_{i} \psi, D \psi, C \psi, \bigcirc \psi$, or $\psi_{1} \mathcal{U} \psi_{2}$ contains at most one free variable.

The monodic fragments of a number of first-order modal logics have been thoroughly investigated $[22,25,15,13,24]$. In the case of $\mathcal{L}_{m}$ this fragment is quite expressive as it contains formulas like the following:

$$
\begin{array}{r}
\forall y(\operatorname{Resource}(y) \rightarrow C(\forall z \operatorname{Available}(y, z) \mathcal{U} \exists x \text { Request }(x, y)) \\
D \bigcirc \forall x y z(\operatorname{Request}(x, y) \rightarrow \neg \text { Available }(y, z)) \rightarrow \\
\rightarrow \bigcirc D \forall x y z(\operatorname{Request}(x, y) \rightarrow \neg \text { Available }(y, z))
\end{array}
$$

According to (1), it is common knowledge that every resource will eventually be requested, but until that time the resource is universally available. By (2) if it is distributed knowledge that at the next step any resource is not available whenever it is requested, then at the next step it is distributed knowledge that this is the case.

Note that the monodic fragment of $\mathcal{L}_{m}$ contains all de dicto formulas, i.e., formulas where no free variable appears in the scope of modal operators, as in (2). So, the limitation is really only on de re formulas.

\section{Message Passing Systems}

In this section we model message passing systems $[4,17]$ in the framework of QIS. A message passing system (MPS) is a MAS in which the only actions for the agents are sending and receiving messages. This setting is common to a variety of distributed systems, well beyond the realms of MAS and AI.

To define our message passing QIS we introduce a set $M s g$ of messages $\mu_{1}, \mu_{2}, \ldots$, and define the local state $l_{i}$ for agent $i$ as a history over $M s g$, that is, a 
sequence of events of the form $\operatorname{send}(i, j, \mu)$ and $\operatorname{rec}(i, j, \mu)$, for $i, j \in A, \mu \in M s g$. Intuitively, $\operatorname{send}(i, j, \mu)$ represents the event where agent $i$ sends to $j$ message $\mu$, while the meaning of $\operatorname{rec}(i, j, \mu)$ is that agent $i$ receives from $j$ message $\mu$. A global state $s \in \mathcal{S}$ is a tuple $\left\langle l_{e}, l_{1}, \ldots, l_{n}\right\rangle$, where $l_{1}, \ldots, l_{n}$ are local states as above, and $l_{e}$ contains all the events in $l_{1}, \ldots, l_{n}$.

A run $r$ over $\langle\mathcal{S}, \mathbb{N}\rangle$ is a function from the natural numbers $\mathbb{N}$ to $\mathcal{S}$ such that:

MP1 $r_{i}(0)$ is a sequence of length zero, and $r_{i}(m+1)$ is either identical to $r_{i}(m)$ or results from appending an event to $r_{i}(m)$.

By MP1 the local states of each agent record the messages she has sent or received, and at each step at most a single event occurs to any agent. We define message passing QIS (MPQIS) as the class of quantified interpreted systems $\mathcal{P}=\langle\mathcal{R}, \mathcal{D}, I\rangle$, where $\mathcal{R}$ is a non-empty set of runs satisfying MP1, $\mathcal{D}$ contains the agents in $A$ and the messages in $M s g$, and $I$ is an interpretation for $\mathcal{L}_{m}$. We use the same notation for objects in the model and syntactic elements.

For the specification of MPS we introduce a predicative letter Send such that $\left(\mathcal{P}^{\sigma}, r, n\right) \models \operatorname{Send}(i, j, \mu)$ iff event $\operatorname{send}(i, j, \mu)$ occurs to agent $i$ at time $n$ in run $r$, i.e., $r_{i}(n)$ is the result of appending $\operatorname{send}(i, j, \mu)$ to $r_{i}(n-1)$. Also, we introduce the predicate Sent such that $\left(\mathcal{P}^{\sigma}, r, n\right) \models \operatorname{Sent}(i, j, \mu)$ iff event $\operatorname{send}(i, j, \mu)$ occurs to agent $i$ before time $n$ in run $r$, i.e., $\operatorname{send}(i, j, \mu)$ appears in $r_{i}(n)$. The predicates Rec and Rec'ed are similarly defined for event $\operatorname{rec}(i, j, \mu)$.

Let us now explore the range of specifications that can be expressed in this formalism. A property often required in MPS is channel reliability. We express this by stating that every sent message is eventually received. Notice that according to the definition of message passing QIS, it is possible that a message is lost during a run of the system. We can force channel reliability by requiring the following specification on MPQIS:

$$
\forall \mu\left(\exists i j \operatorname{Send}(i, j, \mu) \rightarrow F \exists i^{\prime} j^{\prime} \operatorname{Rec}\left(j^{\prime}, i^{\prime}, \mu\right)\right)
$$

In fact, we can be more specific and require that every message is received at most (at least) $k$ steps after being sent, or exactly $k$ steps after being sent:

$$
\begin{array}{r}
\forall \mu\left(\exists i j \operatorname{Send}(i, j, \mu) \rightarrow \bigcirc^{k} \exists i^{\prime} j^{\prime} \operatorname{Rec} \operatorname{ed}\left(j^{\prime}, i^{\prime}, \mu\right)\right) \\
\forall \mu\left(\exists i j \operatorname{Sent}(i, j, \mu) \rightarrow \bigcirc^{k} \exists i^{\prime} j^{\prime} \operatorname{Rec}\left(j^{\prime}, i^{\prime}, \mu\right)\right) \\
\forall \mu\left(\exists i j \operatorname{Send}(i, j, \mu) \rightarrow \bigcirc^{k} \exists i^{\prime} j^{\prime} \operatorname{Rec}\left(j^{\prime}, i^{\prime}, \mu\right)\right)
\end{array}
$$

Note that all of (3)-(6) are monodic. In these specifications the identities of the sender and the receiver are left unspecified. So, in cases in which we are not interested in singling out the addresser and the addressee, the monodic fragment suffices.

Another property often required on MPQIS is that there are no "ghost" messages: if agent $i$ receives a message $\mu$, then $i$ knows that $\mu$ must actually have been sent by some agent $j$. This specification is expressible as a monodic formula:

$$
\forall \mu\left(\exists j \operatorname{Rec} \operatorname{ed}^{\prime}(i, j, \mu) \rightarrow K_{i} \exists j^{\prime} \operatorname{Sent}\left(j^{\prime}, i, \mu\right)\right)
$$


We compare (7) with a further relevant property of MPQIS, i.e., authentication: if agent $i$ has received a message $\mu$ from agent $j$, then $i$ knows that $\mu$ had actually been sent by $j$. This specification can be expressed as the de re version of (7):

$$
\forall \mu j\left(\operatorname{Rec}{ }^{\prime} e d(i, j, \mu) \rightarrow K_{i} \operatorname{Sent}(j, i, \mu)\right)
$$

Note that differently from (7), (8) is not monodic.

Even if we allow an agent $i$ not to know whether a received message $\mu$ has actually been sent, that is, we reject (7), by definition of MPQIS it is distributed knowledge that a message $\mu$ has been sent and received as soon as it has been received, i.e., the following monodic formula holds:

$$
\forall \mu\left(\exists i j \operatorname{Rec}{ }^{\prime} e d(i, j, \mu) \rightarrow D \exists i^{\prime} j^{\prime}\left(\operatorname{Sent}\left(j^{\prime}, i^{\prime}, \mu\right) \wedge \operatorname{Rec} \operatorname{ed}^{\prime}\left(i^{\prime}, j^{\prime}, \mu\right)\right)\right)
$$

On the other hand, the corresponding formula

$$
\forall \mu\left(\exists j \operatorname{Rec} \operatorname{ed}^{\prime}(i, j, \mu) \rightarrow K_{i} \exists j^{\prime}\left(\operatorname{Sent}\left(j^{\prime}, i, \mu\right) \wedge \operatorname{Rec} \operatorname{ed}^{\prime}\left(i, j^{\prime}, \mu\right)\right)\right)
$$

is not valid for any agent $i$.

Furthermore, in $\mathcal{L}_{m}^{1}$ we can express that an agent $i$ cannot aquire the knowledge that message $\mu$ has been sent to her, other than by receiving the message:

$$
\forall \mu\left(\exists j \operatorname{Sent}(j, i, \mu) \rightarrow\left(\neg K_{i} \exists j^{\prime} \operatorname{Sent}\left(j^{\prime}, i, \mu\right) \mathcal{U} \exists j^{\prime \prime} \operatorname{Rec}\left(i, j^{\prime \prime}, \mu\right)\right)\right.
$$

Finally, we might want to check whether at a certain point in the evolution of the MPQIS it will be common knowledge that a message has been sent or received:

$$
\begin{array}{r}
\forall \mu\left(\exists i j \operatorname{Sent}(i, j, \mu) \rightarrow F C\left(\exists i^{\prime} j^{\prime} \operatorname{Sent}\left(i^{\prime}, j^{\prime}, \mu\right)\right)\right) \\
\forall \mu\left(\exists i j \operatorname{Rec} \operatorname{ed}(i, j, \mu) \rightarrow F C\left(\exists i^{\prime} j^{\prime} \operatorname{Rec} \operatorname{ed}\left(i^{\prime}, j^{\prime}, \mu\right)\right)\right)
\end{array}
$$

From results in [4] regarding the attainability of common knowledge in systems with unreliable communication, we may infer that some assumption on channel reliability in MPQIS is needed in order to satisfy specifications (9) and (10). The conclusion we can draw from the observations above is that the monodic fragment of the language $\mathcal{L}_{m}$ allows for rich specifications on MPS, notwithstanding the limitation on free variables in modal contexts.

\section{Axiomatisation}

In this section we present a sound and complete axiomatisation of the set of monodic validities in the class of quantified interpreted systems. The system $Q K T_{m}^{1}$ is a first-order multi-modal version of the propositional epistemic system $S 5$ combined with the linear temporal logic $L T L$.

Definition 5. The system $Q K T_{m}^{1}$ on $\mathcal{L}_{m}^{1}$ contains the following schemes of axioms and inference rules, where $\square$ is any of the epistemic operators $K_{i}$, for $i \in A, D$, or $C$, and $\phi, \psi$ and $\chi$ are formulas in $\mathcal{L}_{m}^{1}$ : 


\begin{tabular}{|l|l|}
\hline Taut & classic propositional tautologies \\
$M P$ & $\phi \rightarrow \psi, \phi \Rightarrow \psi$ \\
\hline$K_{\bigcirc}$ & $\bigcirc \phi \rightarrow \psi) \rightarrow(\bigcirc \phi \rightarrow \bigcirc \psi)$ \\
& $\bigcirc \neg \phi \leftrightarrow \neg \bigcirc \phi$ \\
& $\phi \mathcal{U} \psi \leftrightarrow \psi \vee(\phi \wedge(\phi \mathcal{U} \psi))$ \\
$N e{ }_{\bigcirc}$ & $\phi \Rightarrow \bigcirc \phi$ \\
& $\chi \rightarrow \neg \psi \wedge \bigcirc \chi \Rightarrow \chi \rightarrow \neg(\phi \mathcal{U} \psi)$ \\
\hline$K_{\square}$ & $\square(\phi \rightarrow \psi) \rightarrow(\square \phi \rightarrow \square \psi)$ \\
$T$ & $\square \phi \rightarrow \phi$ \\
4 & $\square \phi \rightarrow \square \square \phi$ \\
5 & $\neg \square \phi \rightarrow \square \neg \square$ \\
$N e c_{\square}$ & $\phi \Rightarrow \square \phi$ \\
\hline & $K_{i} \phi \rightarrow D \phi$ \\
& $C \phi \leftrightarrow(\phi \wedge E C \phi)$ \\
& $\phi \rightarrow(\psi \wedge E \phi) \Rightarrow \phi \rightarrow C \psi$ \\
\hline$B F_{\bigcirc}$ & $\bigcirc \forall x \phi \leftrightarrow \forall x \bigcirc \phi$ \\
$B F_{\square}$ & $\square \forall x \phi \leftrightarrow \forall x \square \phi$ \\
$E x$ & $\forall x \phi \rightarrow \phi[x / t]$ \\
$G e n$ & $\phi \rightarrow \psi[x / t] \Rightarrow \phi \rightarrow \forall x \psi$, for $x$ not free in $\phi$ \\
\hline
\end{tabular}

The operators $K_{i}, D$ and $C$ are $S 5$ modalities, while the next $\bigcirc$ and until $\mathcal{U}$ operators are axiomatised as linear-time modalities. To this we add the classic postulates Ex and Gen for quantification. We consider the standard definitions of proof and theorem: $\vdash \phi$ means that $\phi \in \mathcal{L}_{m}^{1}$ is a theorem in $Q K T_{m}^{1}$.

It is easy to check that the axioms of $Q K T_{m}^{1}$ are valid on every QIS and the inference rules preserve validity. As a consequence, we have the following soundness result:

Theorem 1 (Soundness). The system $Q K T_{m}^{1}$ is sound with respect to the class $\mathcal{Q I S}$ of quantified interpreted systems.

Thus, $Q K T_{m}^{1}$ is sound also for the class $\mathcal{Q} \mathcal{I} \mathcal{S}^{\text {sync }}$ of synchronous QIS.

\subsection{Kripke Models}

To prove the completeness of $Q K T_{m}^{1}$ with respect to $\mathcal{Q} \mathcal{S}$ we first introduce an appropriate class of Kripke models, and prove completeness for these models. Then we apply a correspondence result between Kripke models and QIS.

Definition 6. A Kripke model for $\mathcal{L}_{m}$ is a tuple $\mathcal{M}=\left\langle\left\langle\mathbb{N}_{j},<_{j}\right\rangle_{j \in J},\left\{\sim_{i}\right\}_{i \in A}, \mathcal{D}\right.$, $I\rangle$ such that (i) each $\mathbb{N}_{j}$ is a copy of the naturals with the strict total order $<_{j}$; (ii) for $i \in A, \sim_{i}$ is an equivalence relation on $\bigcup_{j \in J} \mathbb{N}_{j}$; (iii) $\mathcal{D}$ is a non-empty set of individuals; (iv) the interpretation $I$ is such that $I(c) \in \mathcal{D}$, and for $n_{j} \in \mathbb{N}_{j}$, $I\left(P^{k}, n_{j}\right)$ is a k-ary relation on $\mathcal{D}$.

The class of all Kripke models is denoted by $\mathcal{K}$.

A Kripke model is synchronous if for all $i \in A, n_{j} \in \mathbb{N}_{j}, n_{j} \sim_{i} n_{j^{\prime}}^{\prime}$ implies $n=n^{\prime} . \mathcal{K}^{\text {sync }}$ is the class of all synchronous Kripke models. Further, let $R^{*}$ be the reflexive and transitive closure of a given relation $R$. The satisfaction relation $\models$ for an assignment $\sigma$ is inductively defined as follows: 


$$
\begin{array}{ll}
\left(\mathcal{M}^{\sigma}, n_{j}\right) \models P^{k}\left(t_{1}, \ldots, t_{k}\right) & \text { iff }\left\langle I^{\sigma}\left(t_{1}\right), \ldots, I^{\sigma}\left(t_{k}\right)\right\rangle \in I\left(P^{k}, n_{j}\right) \\
\left(\mathcal{M}^{\sigma}, n_{j}\right) \models \neg \psi & \text { iff }\left(\mathcal{M}^{\sigma}, n_{j}\right) \not \psi \\
\left(\mathcal{M}^{\sigma}, n_{j}\right) \models \psi \rightarrow \psi^{\prime} & \text { iff }\left(\mathcal{M}^{\sigma}, n_{j}\right) \not \psi \text { or }\left(\mathcal{M}^{\sigma}, n_{j}\right) \models \psi^{\prime} \\
\left(\mathcal{M}^{\sigma}, n_{j}\right) \models \forall x \psi & \text { iff for all } a \in \mathcal{D},\left(\mathcal{M}^{\sigma}\left(\begin{array}{l}
x \\
a
\end{array}\right), n_{j}\right) \models \psi \\
\left(\mathcal{M}^{\sigma}, n_{j}\right) \models \bigcirc \psi & \text { iff }\left(\mathcal{M}^{\sigma}, n+1_{j}\right) \models \psi \\
\left(\mathcal{M}^{\sigma}, n_{j}\right) \models \psi \mathcal{U} \psi^{\prime} & \text { iff there is } n_{j}^{\prime} \geq_{j} n_{j} \text { such that }\left(\mathcal{M}^{\sigma}, n_{j}^{\prime}\right) \models \psi^{\prime} \\
& \quad \text { and for all } n_{j}^{\prime \prime}, n_{j} \leq_{j} n_{j}^{\prime \prime}<_{j} n_{j}^{\prime} \text { implies }\left(\mathcal{M}^{\sigma}, n_{j}^{\prime \prime}\right) \models \psi \\
\left(\mathcal{M}^{\sigma}, n_{j}\right) \models K_{i} \psi & \text { iff for all } n_{j^{\prime}}^{\prime}, n_{j} \sim_{i} n_{j^{\prime}}^{\prime} \text { implies }\left(\mathcal{M}^{\sigma}, n_{j^{\prime}}^{\prime}\right) \models \psi \\
\left(\mathcal{M}^{\sigma}, n_{j}\right) \models D \psi & \text { iff for all } n_{j^{\prime}}^{\prime},\left(n_{j}, n_{j^{\prime}}^{\prime}\right) \in \bigcap_{i \in A} \sim_{i} \text { implies }\left(\mathcal{M}^{\sigma}, n_{j^{\prime}}^{\prime}\right)=\psi \\
\left(\mathcal{M}^{\sigma}, n_{j}\right) \models C \psi & \text { iff for all } n_{j^{\prime}}^{\prime},\left(n_{j}, n_{j^{\prime}}^{\prime}\right) \in\left(\bigcup_{i \in A} \sim_{i}\right)^{*} \operatorname{implies~}\left(\mathcal{M}^{\sigma}, n_{j^{\prime}}^{\prime}\right) \models \psi
\end{array}
$$

We compare Kripke models and quantified interpreted systems by means of a map $g: \mathcal{K} \rightarrow \mathcal{Q I S}$. Let $\mathcal{M}=\left\langle\left\langle\mathbb{N}_{j},<_{j}\right\rangle_{j \in J},\left\{\sim_{i}\right\}_{i \in A}, \mathcal{D}, I\right\rangle$ be a Kripke model. For every equivalence relation $\sim_{i}$, for $n_{j} \in \mathbb{N}_{j}$, let the equivalence class $\left[n_{j}\right]_{\sim_{i}}=\left\{n_{j^{\prime}}^{\prime} \mid n_{j} \sim_{i} n_{j^{\prime}}^{\prime}\right\}$ be a local state for agent $i$, while $\mathbb{N}_{j}$ is the set of local states for the environment. Then define $g(\mathcal{M})$ as the tuple $\left\langle\mathcal{R}, \mathcal{D}, I^{\prime}\right\rangle$ where $\mathcal{R}$ contains the runs $r_{j}$ such that $r_{j}(n)=\left\langle n_{j},\left[n_{j}\right]_{\sim_{1}}, \ldots,\left[n_{j}\right]_{\sim_{m}}\right\rangle, \mathcal{D}$ is the same as in $\mathcal{M}$, and $I^{\prime}\left(P^{k}, r_{j}, n\right)=I\left(P^{k}, n_{j}\right)$. The structure $g(\mathcal{M})$ is a QIS that satisfies the following result:

Lemma 1. For every $\phi \in \mathcal{L}_{m}, n \in \mathbb{N}$,

$$
\left(\mathcal{M}^{\sigma}, n_{j}\right) \models \phi \text { iff }\left(g(\mathcal{M})^{\sigma}, r_{j}, n\right) \models \phi
$$

We omit the proof of this lemma for reasons of space. Note that if $\mathcal{M}$ is synchronous, then also $g(\mathcal{M})$ is synchronous, i.e., $g: \mathcal{K}^{\text {sync }} \rightarrow \mathcal{Q I \mathcal { S }}{ }^{\text {sync }}$.

\section{Completeness}

In this section we outline the main steps in the completeness proof; we refer to the appendix for definitions and detailed proofs.

The completeness of the system $Q K T_{m}^{1}$ with respect to the class $\mathcal{Q} \mathcal{I}$ of quantified interpreted systems is proved by means of a quasimodel construction [5]. In particular, the version of quasimodels here considered combines the purely epistemic structures in [22] and the purely temporal structures in [25]. As the first step we show that for monodic formulas satisfability in quasimodels implies satisfability in Kripke models.

Lemma 2. If there is a quasimodel $\mathfrak{Q}$ for a monodic formula $\phi \in \mathcal{L}_{m}^{1}$, then $\phi$ is satisfiable in a Kripke model.

Note that if the quasimodel $\mathfrak{Q}$ for $\phi$ is synchronous, then also the Kripke model built from $\mathfrak{Q}$ in Lemma 2 is synchronous.

Next we prove the existence of such a quasimodel for $\phi$.

Lemma 3. Suppose that $\phi \in \mathcal{L}_{m}^{1}$ is a consistent monodic formula, then there exists a (synchronous) quasimodel for $\phi$.

In the proof of Lemma 3 we make use of results in $[22,25]$ regarding purely epistemic and temporal first-order logic. By combining Lemmas 3 and 2 we can state the main result of this paper. 
Theorem 2 (Completeness). The system $Q K T_{m}^{1}$ is complete with respect to the class $\mathcal{Q I S}$ of quantified interpreted systems.

Assume that $\nvdash \phi$, then $\neg \phi$ is consistent and by Lemmas 3 and 2 there is a Kripke model $\mathcal{M}$ satisfying $\neg \phi$. By Lemma 1 the QIS $g(\mathcal{M})$ does not validate $\phi$, therefore $\mathcal{Q I S} \not \models \phi$. Similarly, we can prove the following result.

Theorem 3 (Completeness). The system $Q K T_{m}^{1}$ is complete with respect to the class $\mathcal{Q I S}^{\text {sync }}$ of synchronous QIS.

We refer to the appendix for the details of the proofs.

\section{Conclusions and Further Work}

In this paper we analysed a quantified version of interpreted systems, and proved completeness for the system $Q K T_{m}^{1}$ defined on the monodic fragment of the firstorder language $\mathcal{L}_{m}$, which includes linear-time modalities and epistemic operators for group knowledge. This result makes use of previous contributions on the axiomatisation of pure first-order epistemic and temporal logic [22, 25]. Further, we showed that a wide range of specifications on message passing systems can be expressed in the monodic fragment of $\mathcal{L}_{m}$.

Still, further work is needed in this line of research. The present paper deals with the class $\mathcal{Q} \mathcal{S}$ of all quantified interpreted systems and the class $\mathcal{Q} \mathcal{I} \mathcal{S}^{\text {sync }}$ of synchronous QIS. In the axiomatisation $Q K T_{m}^{1}$ for these classes there is no interaction between temporal and epistemic operators, but interaction is essential to express epistemic concepts such as perfect recall and no learning. These refinements have been widely studied at the propositional level $[7,9]$, but it is not clear to which extent these results apply to the first order. By results in [10] the set of validities in $\mathcal{L}_{m}^{1}$ on the class of QIS with perfect recall is not axiomatisable, as we have unaxiomatisability already at the propositional level. However, to our knowledge there is no result about the monodic fragment in the case that we drop either the linear-time modalities $\bigcirc$ and $\mathcal{U}$, and retain only $F$ and $G$, or the common knowledge operator $C$. Results along this line would be of interest for the investigation of the expressive power of modal logic between propositional and full first-order.

Finally, another issue not tackled in this paper is decidability. We believe that by combining the techniques in $[15,24]$ it is likely to find decidable monodic fragments of first-order temporal epistemic logic. However, this topic demands further investigations.

Acknowledgements: The research described in this paper was partly supported by the EC Framework 6 funded project CONTRACT (IST Project Number 034418), by the research project "Logica Modale e Conoscenza" funded by the Scuola Normale Superiore, Pisa, and by the Royal Society through an International Joint Project award to both authors. 


\section{References}

1. F. Belardinelli, A. Lomuscio. A Complete First-Order Logic of Knowledge and Time. Proceedings of the 11th International Conference on Principles of Knowledge Representation and Reasoning (KR08). pp. 705-714, AAAI Press, 2008.

2. F. Belardinelli, A. Lomuscio. Quantified Epistemic Logics for Reasoning about Knowledge in Multi-Agent Systems. Artificial Intelligence. To appear. Available on-line at http://dx.doi.org/10.1016/j.artint.2009.02.003.

3. P. Blackburn, J. van Benthem, F. Wolter (eds). Handbook of Modal Logic, Elsevier, 2006.

4. R. Fagin, J. Halpern, Y. Moses, M. Vardi. Reasoning about Knowledge, MIT Press, 1995.

5. D. Gabbay, A. Kurucz, F. Wolter, M. Zakharyaschev. Many-Dimensional Modal Logics, Elsevier, 2003.

6. P. Gammie, R. van der Meyden. MCK: Model Checking the Logic of Knowledge, Proceedings of the 16th International Conference on Computer Aided Verification (CAV04), pp. 479-483, Springer, 2004.

7. J. Halpern, R. van der Meyden, M. Vardi. Complete axiomatisations for reasoning about knowledge and time. SIAM Journal on Computing, 33(3):674-703, 2003.

8. J. Halpern, Y. Moses. Knowledge and common knowledge in a distributed environment. Journal of the ACM, 37(3):549-587, 1990.

9. J. Halpern, Y. Moses. A guide to completeness and complexity for modal logics of knowledge and belief. Artificial Intelligence, 54:319-379, 1992.

10. J. Halpern, M. Vardi. The complexity of reasoning about knowledge and time 1: lower bounds. Journal of Computer and System Sciences, 38(1):195-237, 1989.

11. I. Hodkinson. Monodic packed fragment with equality is decidable. Studia Logica, 72:185-197, 2002.

12. I. Hodkinson. Complexity of monodic guarded fragments over linear and real time. Annals of Pure and Applied Logic, 138:94-125, 2006.

13. I. Hodkinson, R. Kontchakov, A. Kurucz, F. Wolter, M. Zakharyaschev. On the computational complexity of decidable fragments of first-order linear temporal logics. Proceedings of the International Symposium on Temporal Representation and Reasoning (TIME03), pp. 91-98, IEEE Press, 2003.

14. I. Hodkinson, F. Wolter, M. Zakharyaschev. Decidable and undecidable fragments of first-order branching temporal logics. Logic in Computer science(LICS02), pp. 393-402, IEEE Computer Society, 2002.

15. I. Hodkinson, F. Wolter, M. Zakharyaschev. Decidable fragment of first-order temporal logics. Annals of Pure and Applied Logic, 106(1-3):85-134, 2000.

16. W. van der Hoek, J.-J. Ch. Meyer, J. Treur. Formal semantics of temporal epistemic reflection. Proceedings of the 4 th International Workshops on Meta-Programming in Logic (META94), pp. 332-352, Springer, 1994.

17. L. Lamport. Time, clocks, and the ordering of events in a distributed system. Communications of the ACM, 21(7):558-565, 1978.

18. R. van der Meyden. Axioms for knowledge and time in distributed systems with perfect recall. Logic in Computer Science (LICS94), pp. 448-457, IEEE, 1994.

19. J.-J. Ch. Meyer, W. van der Hoek. Epistemic Logic for AI and Computer Science. Cambridge University Press, 1995.

20. F. Raimondi, A. Lomuscio. Automatic verification of multi-agent systems by model checking via OBDDs. Journal of Applied Logic, 5(2), pp. 235-251, 2007.

21. M. Reynolds. Axiomatising first-order temporal logic: until and since over linear time. Studia Logica, 57(2/3):279-302, 1996. 
22. H. Sturm, F. Wolter, M. Zakharyaschev. Monodic epistemic predicate logic. Proceedings of the European Workshop on Logics in Artificial Intelligence (JELIA00), pp. 329-344, Springer, 2000.

23. H. Sturm, F. Wolter, M. Zakharyaschev. Common knowledge and quantification. Economic Theory, 19:157-186, 2002.

24. F. Wolter, M. Zakharyaschev. Decidable fragments of first-order modal logics. Journal of Symbolic Logic, 66(3):1415-1438, 2001.

25. F. Wolter, M. Zakharyaschev. Axiomatizing the monodic fragment of first-order temporal logic. Annals of Pure and Applied Logic, 118(1-2):133-145, 2002.

26. F. Wolter. First order common knowledge logics. Studia Logica, 65(2):249-271, 2000 .

27. M. Wooldridge. Computationally grounded theories of agency. Proceedings of the International Conference on Multi-Agent Systems (ICMAS00), pp. 13-22, IEEE Press, 2000.

28. M. Wooldridge. Reasoning about Rational Agents, MIT Press, 2000.

\section{A Appendix}

In this appendix we provide definitions for the main concepts used in the completeness proof for the system $Q K T_{m}^{1}$, as well as the relevant partial results. Intuitively, a quasimodel for a monodic formula $\phi \in \mathcal{L}_{m}^{1}$ is a relational structure whose points are sets of sets of subformulas of $\phi$. Each set of sets of subformulas describes a "possible state of affairs", and contains sets of subformulas defining the individuals in the point.

Definition 7. Given a formula $\phi \in \mathcal{L}_{n}^{1}$, we denote by sub $\phi$ the set of subformulas of $\phi$, and define $s u b_{C} \phi$ as sub $\phi \cup\{E C \psi \mid C \psi \in \operatorname{sub} \phi\} \cup\left\{K_{i} C \psi \mid C \psi \in\right.$ sub $\phi, i \in A\}$. Further, let $s u b_{C} \bigcirc \phi$ be the set $\operatorname{sub}_{C} \phi \cup\left\{\neg \psi \mid \psi \in s u b_{C} \phi\right\} \cup\{\bigcirc \psi \mid$ $\left.\psi \in s u b_{C} \phi\right\} \cup\left\{\bigcirc \neg \psi \mid \psi \in s u b_{C} \phi\right\}$.

Let $s u b_{n} \phi$ be the subset of $s u b_{C} \bigcirc \phi$ containing formulas with at most $n$ free variables, and let $x$ be a variable not occurring in $\phi$, we define $s u b_{x} \phi$ as $\left\{\psi[x / y] \mid \psi[y] \in s u b_{1} \phi\right\}$. Clearly, $x$ is the only free variable in $s u b_{x} \phi$. By con $\phi$ we denote the set of all constants occurring in $\phi$.

Definition 8 (Type). A type for $\phi$ is any subset $\mathfrak{t}$ of sub $b_{x} \phi$ such that for every $\psi, \chi \in s u b_{x} \phi$, (i) $\psi \wedge \chi \in \mathfrak{t}$ iff $\psi \in \mathfrak{t}$ and $\chi \in \mathfrak{t} ;$ (ii) $\neg \psi \in \mathfrak{t}$ iff $\psi \notin \mathfrak{t}$.

This definition of type is completely standard [5, 22, 25]. Two types $\mathfrak{t}, \mathfrak{t}^{\prime}$ agree on $s u b_{0} \phi$ iff $\mathfrak{t} \cap s u b_{0} \phi=\mathfrak{t}^{\prime} \cap s u b_{0} \phi$. Given a type $\mathfrak{t}$ for $\phi$ and a constant $c \in \operatorname{con} \phi$, the pair $\langle\mathfrak{t}, c\rangle$ is called an indexed type for $\phi$.

Also the following definition of state candidate is standard.

Definition 9 (State Candidate). Let $T$ be a set of types for $\phi$ that agree on $s u b_{0} \phi$, and $T^{\text {con }}$ a set containing for each $c \in$ con $\phi$ an indexed type $\langle\mathfrak{t}, c\rangle$ such that $\mathfrak{t} \in T$, then the pair $\mathfrak{C}=\left\langle T, T^{\text {con }}\right\rangle$ is a state candidate for $\phi$.

Given a state candidate $\mathfrak{C}=\left\langle T, T^{c o n}\right\rangle$ we define formula $\alpha_{\mathfrak{C}}$ as follows:

$$
\alpha_{\mathfrak{C}}:=\bigwedge_{\mathfrak{t} \in T} \exists x \mathfrak{t}[x] \wedge \forall x \bigvee_{\mathfrak{t} \in T} \mathfrak{t}[x] \wedge \bigwedge_{\langle\mathfrak{t}, c\rangle \in T^{c o n}} \mathfrak{t}[x / c]
$$


A state candidate $\mathfrak{C}$ is consistent iff the formula $\alpha_{\mathfrak{C}}$ is consistent with $Q K T_{m}^{1}$; consistent state candidates will be the points of our quasimodel. We now define a relation of suitability for types and state candidates which constitute the relational part of our quasimodel.

Definition 10. 1. A pair $\left(\mathfrak{t}_{1}, \mathfrak{t}_{2}\right)$ of types is $\bigcirc$-suitable iff the formula $\mathfrak{t}_{1} \wedge \bigcirc \mathfrak{t}_{2}$ is consistent. It is $i$-suitable iff the formula $\mathfrak{t}_{1} \wedge \neg K_{i} \neg \mathfrak{t}_{2}$ is consistent, and it is $D$-suitable iff the formula $\mathfrak{t}_{1} \wedge \neg D \neg \mathfrak{t}_{2}$ is consistent

2. A pair of state candidates $\left(\mathfrak{C}_{1}, \mathfrak{C}_{2}\right)$ is $\bigcirc$-suitable iff the formula $\alpha_{\mathfrak{C}_{1}} \wedge \bigcirc \alpha_{\mathfrak{C}_{2}}$ is consistent. It is $i$-suitable iff the formula $\alpha_{\mathfrak{C}_{1}} \wedge \neg K_{i} \neg \alpha_{\mathfrak{C}_{2}}$ is consistent, and it is $D$-suitable iff the formula $\alpha_{\mathfrak{C}_{1}} \wedge \neg D \neg \alpha_{\mathfrak{C}_{2}}$ is consistent.

We now introduce the frame underlying the quasimodel for $\phi$.

Definition 11 (Frame). Let $A^{+}=A \cup\{D\}$. $A$ frame $\mathcal{F}$ is a tuple $\left\langle\left\langle\mathbb{N}_{j},<_{j}\right\rangle_{j \in J},\left\{\prec_{l}\right\}_{l \in A^{+}}\right\rangle$such that (i) each $\mathbb{N}_{j}$ is a copy of the natural numbers with the strict total order $<_{j}$; (ii) the pair $\left\langle\bigcup_{j \in J} \mathbb{N}_{j}, \bigcup_{l \in A^{+}} \prec_{l}\right\rangle$ is a set of disjoint intransitive trees ${ }^{1}$.

A frame is synchronous if for all $l \in A^{+}, n=n^{\prime}$ whenever $n_{j} \prec_{l} n_{j^{\prime}}^{\prime}$. Further, we introduce state functions mapping points in $\mathcal{F}$ to state candidates.

Definition 12 (State Function). A state function for $\phi$ over $\mathcal{F}$ is a map $\mathfrak{f}$ associating with each $n_{j} \in \mathcal{F}$ a consistent state candidate $\mathfrak{f}\left(n_{j}\right)=\mathfrak{C}_{n_{j}}$ for $\phi$ such that (i) the domain of $\mathfrak{f}$ is not empty; (ii) if $\mathfrak{f}$ is defined on $n_{j}$, then $\mathfrak{f}$ is defined on $n+1_{j}$; (iii) if $\mathfrak{f}$ is defined on $n_{j}$ and $n_{j} \prec_{l} n_{j^{\prime}}^{\prime}$ then $\mathfrak{f}$ is defined on $n_{j^{\prime}}^{\prime}$.

This definition of state function takes into account also the case of synchronous systems. In what follows we often do not distinguish between a state $n_{j}$ and its associated state candidate $\mathfrak{f}\left(n_{j}\right)=\mathfrak{C}_{n_{j}}$.

Finally, we provide the definition of objects, which correspond to the runs in $[22,25]$. We choose this name to avoid confusion with the runs in QIS.

Definition 13 (Object). Let $\mathfrak{f}$ be a state function for $\phi$ over $\mathcal{F}$. An object in $\langle\mathcal{F}, \mathfrak{f}\rangle$ is a map $\rho$ associating with every $n_{j} \in \mathbb{N}_{j}$ a type $\rho\left(n_{j}\right)$ in $T_{n_{j}}$ such that

1. the pairs $\left(\rho\left(n_{j}\right), \rho\left(n+1_{j}\right)\right)$ are $\bigcirc$-suitable;

2. $\rho\left(n_{j}\right)$ and $\rho\left(n_{j^{\prime}}^{\prime}\right)$ are l-suitable whenever $n_{j} \prec_{l} n_{j^{\prime}}^{\prime}$;

3. if $\chi \mathcal{U} \psi \in \rho\left(n_{j}\right)$ then there is $n^{\prime} \geq n$ such that $\psi \in \rho\left(n_{j}^{\prime}\right)$ and $\chi \in \rho\left(n_{j}^{\prime \prime}\right)$ for all $n \leq n^{\prime \prime}<n^{\prime}$;

4. if $\neg K_{i} \psi \in \rho\left(n_{j}\right)$ then for some $n_{j^{\prime}}^{\prime}, \rho\left(n_{j}\right) \prec_{i} \rho\left(n_{j^{\prime}}^{\prime}\right)$ and $\psi \notin \rho\left(n_{j^{\prime}}^{\prime}\right)$;

5. if $\neg D \psi \in \rho\left(n_{j}\right)$ then for some $\left.n_{j^{\prime}}^{\prime}, \rho\left(n_{j}\right) \prec_{D} \rho\left(n_{j^{\prime}}^{\prime}\right)\right)$ and $\psi \notin \rho\left(n_{j^{\prime}}^{\prime}\right)$;

6. if $\neg C \psi \in \rho\left(n_{j}\right)$ then for some $n_{j^{\prime}}^{\prime},\left(\rho\left(n_{j}\right), \rho\left(n_{j^{\prime}}^{\prime}\right)\right) \in\left(\bigcup_{l \in A^{+}} \prec_{l}\right)^{*}$ and $\psi \notin$ $\rho\left(n_{j^{\prime}}^{\prime}\right)$.

${ }^{1}$ The pair $\langle U, R\rangle$ is an intransitive tree iff (i) there is a root $u_{0} \in U$ such that $u_{0} R^{*} u$ for every $u \in U$; (ii) for every $u \in U$ the set $\left\{u \in U \mid u R^{*} u\right\}$ is finite and linearly ordered by $R^{*}$; (iii) every $u \in U$ but the root $u_{0}$ has exactly one predecessor; (iv) the root $u_{0}$ is irreflexive. 
A map $\rho$ associating with every $n_{j} \in \mathbb{N}_{j}$ a type $\rho\left(n_{j}\right) \in T_{n_{j}}$ such that only (1) and (3) hold is a temporal object. Similarly, a map $\rho$ associating with every $n_{j} \in \mathbb{N}_{j}$ a type $\rho\left(n_{j}\right) \in T_{n_{j}}$ such that only (2) and (4)-(6) hold is an epistemic object. Now we have all the elements to give the definition of quasimodels.

Definition 14 (Quasimodel). A quasimodel for $\phi$ is a tuple $\mathfrak{Q}=\langle\mathcal{F}, \mathfrak{f}, \mathcal{O}\rangle$ such that $\mathfrak{f}$ is a state function over $\mathcal{F}$, and

1. $\phi \in \mathfrak{t}$, for some $\mathfrak{t} \in T_{n_{j}}$ and $T_{n_{j}} \in \mathfrak{C}_{n_{j}}$

2. every pair $\left(\mathfrak{C}_{n_{j}}, \mathfrak{C}_{n+1_{j}}\right)$ is $\bigcirc$-suitable, and every pair $\left(\mathfrak{C}_{n_{j}}, \mathfrak{C}_{n_{j^{\prime}}^{\prime}}\right)$ is l-suitable whenever $n_{j} \prec_{l} n_{j^{\prime}}^{\prime}$

3. for every $\mathfrak{t} \in T_{n_{j}}$ there exists an object $\rho \in \mathcal{O}$ such that $\rho\left(n_{j}\right)=\mathfrak{t}$

4. for every $c \in$ con $\phi$, the function $\rho_{c}$ such that $\rho_{c}\left(n_{j}\right)=\mathfrak{t}$, for $\langle\mathfrak{t}, c\rangle \in T_{n_{j}}^{\text {con }}$ is an object in $\mathcal{O}$.

We can now prove Lemma 2.

Lemma 2 If there is a quasimodel $\mathfrak{Q}$ for a monodic formula $\phi \in \mathcal{L}_{m}^{1}$, then $\phi$ is satisfiable in a Kripke model.

Proof. The proof of this lemma is similar to those for Lemmas 11.72 and 12.9 in $[5]$.

First of all, for every monodic formula $\psi \in \mathcal{L}_{m}^{1}$ of the form $K_{i} \chi, D \chi, C \chi$, $\bigcirc \chi$, or $\chi_{1} \mathcal{U} \chi_{2}$ we consider a $k$-ary predicate $P_{\psi}^{k}$, for $k$ equal to 0 or 1 . The formula $P_{\psi}^{k}(x)$ is the surrogate of $\psi$. Given a formula $\phi \in \mathcal{L}_{m}^{1}$ we denote by $\bar{\phi}$ the formula obtained from $\phi$ by substituting all its modal subformulas which are not within the scope of another modal operator by their surrogates.

Since every state candidate $\mathfrak{C}$ in the quasimodel $\mathfrak{Q}$ is consistent and the system $Q K T_{m}^{1}$ is based on first-order logic, the formula $\bar{\alpha}_{\mathfrak{C}}$ is consistent with first-order (non-modal) logic. As a consequence, by completeness of first-order logic, there is a first-order structure $\mathcal{I}=\langle I, \mathcal{D}\rangle$, where $\mathcal{D}$ is a non-empty set of individuals and $I$ is an interpretation on $\mathcal{D}$, which satisfies $\bar{\alpha}_{\mathfrak{C}}$, that is, $I^{\sigma}=\bar{\alpha}_{\mathfrak{C}}$ for some assignment $\sigma$ to $\mathcal{D}$.

Now, we consider a cardinal $\kappa \geq \aleph_{0}$ greater than the cardinality of the set $\mathcal{O}$ of all objects in $\mathfrak{Q}$, and define $\mathcal{D}=\{\langle\rho, \xi\rangle \mid \rho \in \mathcal{O}, \xi<\kappa\}$. By the theory of first-order logic, we can assume without loss of generality that $\mathcal{D}$ is the domain of the first-order structure $\mathcal{I}_{n_{j}}=\left\langle I_{n_{j}}, \mathcal{D}\right\rangle$ satisfying $\bar{\alpha}_{\mathfrak{C}_{n_{j}}}$, that is, all structures $\mathcal{I}_{n_{j}}$ share a common domain $\mathcal{D}$, and for every $\mathfrak{t} \in T_{n_{j}},\langle\rho, \xi\rangle \in \mathcal{D}, \rho\left(n_{j}\right)=\mathfrak{t}$ iff $I_{n_{j}}^{\sigma} \models \overline{\mathfrak{t}}[x]$, for $\sigma(x)=\langle\rho, \xi\rangle$. Moreover, $I_{n_{j}}(c)=\langle\rho, 0\rangle$, for every $c \in \operatorname{con} \phi$.

Let us now define the Kripke model $\mathcal{M}$. Let $\mathcal{F}=\left\langle\left\langle\mathbb{N}_{j},<_{j}\right\rangle_{j \in J},\left\{\prec_{l}\right\}_{l \in A^{+}}\right\rangle$ be the frame of the quasimodel $\mathfrak{Q}$, we define $\mathcal{M}$ as $\left\langle\left\langle\mathbb{N}_{j},<_{j}\right\rangle_{j \in J},\left\{R_{i}\right\}_{i \in A}, \mathcal{D}, I\right\rangle$ where each sequence $\mathbb{N}_{j}$ of naturals in $\mathcal{F}$ belongs also to $\mathcal{M}$; each relation $R_{i}$ is the reflexive, symmetric and transitive closure of $\prec_{i} \cup \prec_{D}$; $\mathcal{D}$ is defined as above; and the interpretation $I$ is obtained by gluing together the various $I_{n_{j}}$.

By induction on the length of $\psi \in s u b_{x} \phi$ we can show that for every $\sigma$,

$$
I_{n_{j}}^{\sigma} \models \bar{\psi} \text { iff }\left(\mathcal{M}^{\sigma}, n_{j}\right) \models \psi
$$


The base of induction follows by definition of $I$. The step for propositional connectives and quantifiers follows by the induction hypothesis and equations $\overline{\psi_{1} \rightarrow \psi_{2}}=\overline{\psi_{1}} \rightarrow \overline{\psi_{2}}, \overline{\neg \psi_{1}}=\neg \overline{\psi_{1}}, \overline{\forall x \psi_{1}}=\forall x \overline{\psi_{1}}$. To deal with modal operators we state without proof the following remark, the relevant cases directly follow.

Remark 1. For every $\rho \in \mathcal{O}$ and $n_{j} \in \mathbb{N}_{j}$,

(i) $\bigcirc \psi \in \rho\left(n_{j}\right)$ iff $\psi \in \rho\left(n+1_{j}\right)$

(ii) $\psi \mathcal{U} \chi \in \rho\left(n_{j}\right)$ iff there exists $n_{j}^{\prime} \geq_{j} n_{j}$ such that $\chi \in \rho\left(n_{j}^{\prime}\right)$

and for every $n_{j} \leq_{j} n_{j}^{\prime \prime}<n_{j}^{\prime}, \psi \in \rho\left(n_{j}^{\prime \prime}\right)$

(iii) $K_{i} \psi \in \rho\left(n_{j}\right)$ iff for every $n_{j^{\prime}}^{\prime}, n_{j} R_{i} n_{j^{\prime}}^{\prime}$ implies $\psi \in \rho\left(n_{j^{\prime}}^{\prime}\right)$

(iv) $D \psi \in \rho\left(n_{j}\right)$ iff for every $n_{j^{\prime}}^{\prime},\left(n_{j}, n_{j^{\prime}}^{\prime}\right) \in \bigcap_{i \in A} R_{i}$ implies $\psi \in \rho\left(n_{j^{\prime}}^{\prime}\right)$

$(v) C \psi \in \rho\left(n_{j}\right)$ iff for every $n_{j^{\prime}}^{\prime},\left(n_{j}, n_{j^{\prime}}^{\prime}\right) \in\left(\bigcup_{i \in A} R_{i}\right)^{*}$ implies $\psi \in \rho\left(n_{j^{\prime}}^{\prime}\right)$

The proof of this remark is similar to the one for Lemma 12.10 in [5]. To complete the proof of Lemma 2 we remark that by definition of quasimodel $\phi \in \mathfrak{t}$, for some $\mathfrak{t} \in T_{n_{j}}$ and $T_{n_{j}} \in \mathfrak{C}_{n_{j}}$, therefore we have that $\phi$ is satisfied in the Kripke model $\mathcal{M}$.

Note that if $\mathfrak{Q}$ is a synchronous quasimodel for $\phi$, then the Kripke model built from $\mathfrak{Q}$ in Theorem 2 is also synchronous.

Now it is left to prove the existence of such a quasimodel for $\phi$.

Lemma 3 Suppose that $\phi \in \mathcal{L}_{m}^{1}$ is a consistent monodic formula, then there exists a (synchronous) quasimodel for $\phi$.

In the proof we use the following partial results. These lemmas, which we state without proof, are modifications of Lemmas 11.73 and 12.11 in [5].

Lemma 4. Let $\mathfrak{C}$ be a consistent state candidate, then we can construct an infinite sequence $\left\{\mathfrak{C}_{n}\right\}_{n \in \mathbb{N}}$ of state candidates such that (i) every pair $\left(\mathfrak{C}_{n}, \mathfrak{C}_{n+1}\right)$ is $\bigcirc$-suitable; (ii) for every $\mathfrak{t} \in T_{n}$ there exists a temporal object $\rho$ such that $\rho(n)=\mathfrak{t}$; (iii) for $c \in$ con $\phi$, the function $\rho_{c}$ such that $\rho_{c}(n)=\mathfrak{t}$, for $\langle\mathfrak{t}, c\rangle \in T_{n}^{c o n}$, is a temporal object.

Lemma 5. Let $\mathfrak{C}$ be a consistent state candidate, then we can construct a structure $\mathcal{W}=\left\langle W, \prec_{1}, \ldots, \prec_{m}, \prec_{D}\right\rangle$ such that $W$ is a non-empty set of state candidates, and the pair $\left\langle W, \bigcup_{l \in A^{+}} \prec_{l}\right\rangle$ is a tree. Further, (i) $\mathfrak{C} \prec_{l} \mathfrak{C}^{\prime}$ only if $\mathfrak{C}$ and $\mathfrak{C}^{\prime}$ are l-suitable; (ii) for every $\mathfrak{t} \in T, w \in W$, there exists an epistemic object $\rho$ such that $\rho(w)=\mathfrak{t}$; (iii) for $c \in$ con $\phi$, the function $\rho_{c}$ such that $\rho_{c}(w)=\mathfrak{t}$, for $\langle\mathfrak{t}, c\rangle \in T_{w}^{c o n}$, is an epistemic object.

We can now prove Lemma 3. 
Proof. Let $\pi_{\phi}$ be the disjunction of all formulas $\alpha_{\mathfrak{C}}$, for all state candidates for $\phi$. Note that $\bar{\pi}_{\phi}$ is true in every first-order model, so by completeness we have that $\vdash \pi_{\phi}$. Since $\phi$ is consistent, also $\phi \wedge \pi_{\phi}$ is consistent. Then there is a consistent state candidate $\mathfrak{C}=\left\langle T, T^{\text {con }}\right\rangle$ such that $\phi \in \mathfrak{t}$, for some $\mathfrak{t} \in T$.

We define the structure $\langle\mathcal{F}, \mathfrak{f}\rangle$ underlying the quasimodel $\mathfrak{Q}$ in steps. At step $2 n+1$ we extend the structure with a chain $\mathbb{N}_{\mathfrak{C}^{\prime}}$ of state candidates for every state candidate $\mathfrak{C}^{\prime}$ introduced at step $2 n$. At stage $2 n+2$ we provide every state candidate introduced at step $2 n+1$ with a tree of state candidates as shown in Lemma 5 .

We start with the base of induction. Define $\mathcal{F}_{0}=\left\langle\left\langle\mathbb{N}_{j},<_{j}\right\rangle_{j \in J_{0}},\left\{\prec_{l}^{0}\right\}_{l \in A^{+}}\right\rangle$, where $J_{0}$ is empty and for every $l \in A^{+}, \prec_{l}^{0}$ is also empty. The function $\mathfrak{f}_{0}$ is empty as well. We also consider a set $U_{0}$ which contains only the state candidate $\mathfrak{C}$ defined above, and assume $U_{-1}=\emptyset$.

At step $2 n+1$ the frame $\mathcal{F}_{2 n+1}$ is defined as the tuple $\left\langle\left\langle\mathbb{N}_{j},<_{j}\right\rangle_{j \in J_{2 n+1}}\right.$, $\left.\left\{\prec_{l}^{2 n+1}\right\}_{l \in A^{+}}\right\rangle$such that $J_{2 n+1}=J_{2 n} \cup\left\{U_{2 n} \backslash U_{2 n-1}\right\}$, and for each $l \in A^{+}$, $\prec_{l}^{2 n+1}=\prec_{l}^{2 n}$. Further, for every $u \in U_{2 n} \backslash U_{2 n-1}$ by Lemma 4 there exists a sequence $\left\{u_{k}\right\}_{k \in \mathbb{N}}$ of state candidates such that $u_{0}=u$. Thus, the state function $\mathfrak{f}_{2 n}$ is extended to $\mathfrak{f}_{2 n+1}$ such that $\mathfrak{f}_{2 n+1}\left(n_{u}\right)=u_{n}$, for $u \in U_{2 n} \backslash U_{2 n-1}$, and $\mathfrak{f}_{2 n+1}$ is equal to $\mathfrak{f}_{2 n}$ on all the other $u$. Finally, $U_{2 n+1}=\bigcup_{j \in J_{2 n+1}} \mathbb{N}_{j}$.

For defining $\mathcal{F}_{2 n+2}$ we take $J_{2 n+2}=J_{2 n+1}$. Moreover, by Lemma 5 for every $u \in U_{2 n+1} \backslash U_{2 n}$ there is a structure $\left\langle W_{u},\left\{\prec_{l}\right\}_{l \in A^{+}}\right\rangle$such that the pair $\left\langle W_{u}, \bigcup_{l \in A^{+}} \prec_{l}\right\rangle$ is a tree. We define $\prec_{l}^{2 n+2}$ as $\prec_{l}^{2 n+1} \cup \prec_{l}$, for each $l \in A^{+}$. Finally, $\mathfrak{f}_{2 n+2}=\mathfrak{f}_{2 n+1}$ and $U_{2 n+2}=U_{2 n+1} \cup \bigcup_{u \in U_{2 n+1} \backslash U_{2 n}} W_{u}$.

Now consider the quasimodel $\mathfrak{Q}=\langle\mathcal{F}, \mathfrak{f}, \mathcal{O}\rangle$, where $\mathcal{F}=\left\langle\left\langle\mathbb{N}_{j},<_{j}\right\rangle_{j \in J}\right.$, $\left.\left\{\prec_{l}\right\}_{l \in A^{+}}\right\rangle$such that $J=\bigcup_{k \in \mathbb{N}} J_{k}$ and $\prec_{l}=\bigcup_{k \in \mathbb{N}} \prec_{l}^{k}$, for $l \in A^{+}, \mathfrak{f}=\bigcup_{k \in \mathbb{N}} \mathfrak{f}_{k}$, and $\mathcal{O}$ is the set of all objects on $\langle\mathcal{F}, \mathfrak{f}\rangle$. By Lemmas 4 and 5 and by construction of $\mathfrak{Q}$ we can show that the objects in $\mathcal{O}$ satisfy the constraints on quasimodels. Since $\phi \in \mathfrak{t}$, for some $\mathfrak{t} \in \mathfrak{C}$ and $\mathfrak{C} \in \mathfrak{Q}$, we have that $\mathfrak{Q}$ is a quasimodel for $\phi$.

Furthermore, if we want to obtain a synchronous quasimodel from the construction above we modify the step $2 n+1$, for $n \geq 1$, as follows. For every $u \in U_{2 n} \backslash U_{2 n-1}$ by construction there exists a structure $\left\langle W_{u^{\prime}},\left\{\prec_{l}\right\}_{l \in A^{+}}\right\rangle$, for some $u^{\prime} \in U_{2 n-1}$, such that $u \in W_{u^{\prime}}$. Moreover, for some $j \in J_{2 n}, m \in \mathbb{N}$, $u^{\prime}=m_{j}$. Now, by Lemma 4 there exists a sequence $\left\{u_{k}\right\}_{k \in \mathbb{N}}$ of state candidates such that $u_{0}=u$, but now define the state function $\mathfrak{f}_{2 n+1}$ such that $\mathfrak{f}_{2 n+1}\left((m+k)_{u}\right)=u_{k}$ for $k \in \mathbb{N}$, where $m$ is as above. It it not difficult to show that by this construction the quasimodel $\mathfrak{Q}$ for $\phi$ is synchronous. This completes the proof of Lemma 2.

From Lemmas 3 and 2, Theorems 2 and 3 follow by Lemma 1. This completes the completeness proof. 